peptide as well as an understanding of structural characteristics that may contribute to TIL1383I's co-receptor independence.

4222

\section{Synthesis and application of cyanuric chloride lipids for} peptide presentation

David Nardo ${ }^{1}$, and Vincent J. Venditto ${ }^{2}$

${ }^{1}$ University of Kentucky Center for Clinical and Translational

Science; ${ }^{2}$ University of Kentucky

OBJECTIVES/GOALS: My long-term career objective is to become an established independent researcher focused on understanding and modulating immune responses to biologics in order to enhance their efficacy and understand the underlying mechanisms by which these interact with the immune system. METHODS/STUDY POPULATION: In this study we will evaluate the utility of cyanuric chloride based synthetic lipids in the presentation of peptide epitopes of the gene delivery vector, adeno-associated virus (AAV). The lipopeptide conjugates will be administered to mice via liposomal formulations to assess their ability to induce immune responses by ELISA as compared to mice treated with the AAV. The three-dimensional conformation of the peptides will be evaluated using nuclear magnetic resonance to determine their similarity with the natural conformation that these peptides adapt on the viral surface. Additionally, to assess the translatability of this conjugation strategy, we will test the ability of our lipopeptides to bind to serum antibodies from human subjects. RESULTS/ANTICIPATED RESULTS: We anticipate that peptide presentation using cyanuric chloride lipids will achieve a robust response in mice following immunization. Immunizations with our lipids should induce the production of antibodies targeting AAV, albeit a milder response that the virus itself, given the complexity of viral epitopes. Nuclear magnetic resonance will inform us on how to improve the synthetic lipids to optimize peptide presentation by altering the characteristics of the lipid anchors. Finally, by using human serum to test for the ability of our lipopeptides to bind to antibodies in serum from patients positive for AAV antibodies, we can become informed on whether our strategy has utility in human studies or whether our method is limited to animal models of human disease. DISCUSSION/ SIGNIFICANCE OF IMPACT: The current work seeks to develop a strategy to present peptides from a well characterized biologic, $\mathrm{AAV}$, on a liposome surface. Our ultimate purpose is to employ liposomal formulations as decoys that target AAV-specific lymphocytes to improve the in vivo efficacy of AAV.

4296

Targeting ERG Through Toll-Like Receptor 4 in Prostate Cancer

Ben Greulich ${ }^{1}$, Josh Plotnik, and Peter Hollenhorst

${ }^{1}$ Indiana University School of Medicine

OBJECTIVES/GOALS: The objective of this research was to learn how the oncogenic transcription factor, ERG, is regulated in prostate cancer. If we could learn how ERG is regulated and which genes are important for its oncogenic phenotype in prostate cells, we could design new therapeutic strategies against ERG, which has proven to be difficult to target. METHODS/STUDY POPULATION: We conducted an shRNA screen in prostate cells to determine candidate genes and pathways that are important for ERG function. To validate the findings of the screen, we performed a variety of cell-based functional assays, including trans-well migration, wound healing, and clonogenic survival assays. To further investigate the mechanism between ERG and the genes revealed by the screen, we performed biochemical and molecular biology experiments such as Western blotting and qRT-PCR for protein and mRNA expression, co-immunoprecipitation assays to determine protein-protein interactions, and chromatin immunoprecipitation (ChIP-qPCR) to determine transcription factor binding to DNA sites. RESULTS/ ANTICIPATED RESULTS: The screen revealed that genes involved in the toll-like receptor 4 (TLR4) pathway are important for ERGmediated migration. We tested the effect of a TLR4 inhibitor on ERG function and observed decreased migration and clonogenic survival exclusively in ERG-positive cells. Expression of pMEK and pERG was reduced when TLR4 was inhibited, which suggests a mechanism in which TLR4 upregulates pMEK, leading to the phosphorylation and activation of ERG. This is supported by functional assays in which cells expressing a phosphomimetic ERG are resistant to the TLR4 inhibitor. We demonstrated that ERG drives the transcription of TLR4 and its endogenous ligands HSPA8 and BGN. Therefore, ERG can sensitize the cell to TLR4 activation by increasing the number of receptors as well as providing the ligands needed for stimulation. DISCUSSION/SIGNIFICANCE OF IMPACT: This research provides a new therapeutic pathway for treating ERG-positive patients through TLR4 inhibition. This can be beneficial because many patients become resistant to the standard therapy, leaving very few treatment options. TLR4-based therapies could provide an alternative for patients who have developed resistance.

4263

The cardioprotective effects of ramipril during the course of experimental hypercholesterolemia in rabbits

Zvezdana Zivorad Kojic ${ }^{1}$

${ }^{1}$ University of Belgrade, School of Medicine

OBJECTIVES/GOALS: High cholesterol is among the major causes of cardiometabolic complications. People with high cholesterol have about twice the risk of heart disease as people with lower levels. Approximately every 40 seconds, an American will have a heart attack. Costs related to Heart Attack exceed 60 Billion USA dollars per year. Renin-Angiotensin-Aldosteron System (RAAS) is implicated in the genesis of coronary heart disease and in the perpetuation of heart failure. Angiotensin-Converting Enzyme inhibitors (ACE-I) have emerged as the treatment of choice for patients with all degrees of heart failure. Many clinical trials (Consensus, 1987; Save 1990) provide the evidence that ACE-I preserves cardiac function, prevents cardiovascular death, myocardial infarction \& stroke and limit remodeling after myocardial infarction. However, there are still controversies in cardiology and a debate over cardioprotection is continuing:

- Do ACE Inhibitors have unique properties, beyond their antihypertensive effect?

- Can we protect the heart during hypercholesterolemia?

- In which way hypercholesterolemia affects mitochondria bioenergetics?

- How does ramipril affect mitochondrial bioenergetics during the course of experimental hypercholesterolemia?

Objectives/Goals were: To evaluate the mitochondrial actions of chronically administered ramipril (non-SH-containing ACE inhibitor) in cholesterol-fed rabbits by determining the influence of ramipril on: 
- myocardial oxygen consumption (State 4, State 3), Respiratory Control Ratio (RCR), and adenosine diphosphate - oxygen index $(\mathrm{ADP} / \mathrm{O})$ and

- oxidative stress biomarkers

METHODS/STUDY POPULATION: Animal treatments. In the course of twelve-week the male Chinchilla rabbits $(n=10$ /group) received once a day a single dose, in group: A - sunflower oil (control animals); B - atherogenic 2\% hypercholesterolemic diet; C - atherogenic diet and ramipril $(1 \mathrm{mg} / \mathrm{kg})$ and $\mathrm{D}$ - ramipril $(1 \mathrm{mg} / \mathrm{kg})$ only. Animals were terminated in accordance with the U.K. "Animals (Scientific Procedures) Act." Isolation of mitochondria Mitochondria from rabbit heart were isolated by tissue digestion (trypsin), fractionation and differential centrifugation. Mitochondrial respiratory functional measures (State 4 - Basal; State 3 - ADP-stimulated respiration, $R C R$ and $A D P / O)$ and biochemical markers of oxidative damage (the nitrite level) were measured polarographically (Clark electrode, YSI, USA) and spectrophotometrically, respectively, in isolated heart mitochondrial suspensions. Statistics - All results are reported as means $\pm S D$. Comparisons between ramipril treated and control animals were performed by unpaired t-test or one-way ANOVA with a Tukey adjustment for multiple comparisons. A $P$ value $<0.05$ was considered significant for all tests. RESULTS/ANTICIPATED RESULTS: Plasma cholesterol levels: After a period of 12 weeks

- Plasma cholesterol levels in control rabbits (A) were low (1.36 \pm $0.23 \mathrm{mmol} / \mathrm{l})$.

- Cholesterol-fed rabbits became hypercholesterolemic and their plasma total cholesterol level was higher even than $10 \mathrm{mmol} / \mathrm{l}$. The level of total cholesterol in the high-cholesterol-diet group was significantly increased compared with the level in the normal-diet group $(\mathrm{p}<0.01)$.

- In the high-cholesterol-diet group treated with ramipril (C), the plasma cholesterol level was not affected by the drug ramipril $(10.54 \pm 1.31 \mathrm{mmol} / \mathrm{l})$. ACE-I ramipril did not infuence the concentration of total cholesterol.

- Plasma cholesterol levels in group D were low (1.46 \pm 0.29 $\mathrm{mmol} / \mathrm{l})$.

Mitochondria protein concentration: 10-15 mg per heart ( $0.5 \mathrm{mg} / \mathrm{ml}$ ). Mitochondrial actions of ACE-I ramipril: a typical mitochondrial oxygen consumption rates in State 4 (basal) and State $3(+\mathrm{ADP})$ in malate-energized heart mitochondria after 12 -week treatment protocol highlights that, comparing to the control group:

- Atherogenic $2 \%$ cholesterol diet (B) caused a decline in mitochondrial function (in both, State 3 and 4) (-25\%).

- Mitochondria from group C animals (treatment with ramipril along with $2 \%$ cholesterol diet) exhibited higher State 3 respiratory rates compared with group $B$.

- Mild inhibition of mitochondrial respiration was recorded in group $\mathrm{D}$, in both respiratory states (V4\&V3).

No significant difference between the groups were found regarding RCR. However, high significant statistical decrease $(\mathrm{p}<0.01)$ was found in group B regarding ADP/O ratio. Nitrite levels: Rabbits receiving a supplementation of $2 \%$ cholesterol for 12 weeks (B) showed an increase in nitrite levels $(115 \pm 21 \mathrm{nmol} / \mathrm{l}$ vs $50 \pm 9$ $\mathrm{nmol} / \mathrm{l}$ controls). However, It was attenuated by ramipril (C) $(85 \pm 16 \mathrm{nmol} / \mathrm{l})$. Strong correlations were found between State
3 respiratory rate and the nitrite level $(r=-.967, p<0.05)$. It means: the higher level of nitrite - the lower oxygen consumption rate. DISCUSSION/SIGNIFICANCE OF IMPACT: The beneficial effect of ramipril was not noticed on plasma cholesterol levels. However, ramipril improves rabbit heart mitochondrial bioenergetics function during the course of experimental hypercholesterolemia.

- In cholestrerol-supplemented hearts myocardial oxygen consumption was markedly reduced (State 4 and State 3) compared to controls. Administration of high-cholesterol diet decreased not only the respiratory activity of rabbit heart mitochondria (RHM), but also the sensitivity of respiratory chain to ADP (ADP/O), while concomitantly caused an increase in nitrite production. Possible explanation: high-fat diet affects the fluidity of mitochondrial membrane - Electron transport chain (ETC) may be damaged, and unable to support high rates of respiration (e.g. substantial cytochrome c could be lost).

- Administration of ACE-I ramipril along with cholesterol diet partially abolish reduction in MQO2 and improved coupling efficiency (ADP/O). Possible explanation: reduced coupling efficiency means the coupling mechanism itself is altered (e.g. the respiratory complexes slip and pump fewer protons than normal and less ATP is produced per oxygen consumed. Ramipril partially improved coupling efficiency and increased the amount of ATP per oxygen consumed.

- RCR - No significant difference between the groups were found. High RCR indicates good function (a high capacity for substrate oxidation and ATP turnover). Low RCR usually indicates dysfunction. However, there is no absolute RCR value that is diagnostic of dysfunctional mitochondria, because values are substrate- and tissue-dependent.

- NO• exerts metabolic control over mitochondrial respiration. Group B: The lowered state 3-respiration in heart mitochondria seems to contribute to the increased NO production, and elevated nitrite level.

- In a system as complex as OXPHOS, conclusions about overall efficiency must involve measurements of: mito membrane potential, proton transport, ATP synthesis and modular kinetic analisys.

Measurements both, respiration \& potentials will enable us to identify the primary site of effectors, allowing deeper quantitative analyses and better patients therapy.

4371

\section{The Role of B Cells in Keloid Formation}

Jaclyn B Anderson ${ }^{1}$, Alexander B Harrant, Nalu Navarro-Alvarez, Zhaohui Wang, Adrie van Bokhoven, Whitney High, Tae W Chong, and Christene A. Huang

${ }^{1}$ University of Colorado at Denver

OBJECTIVES/GOALS: Recent studies indicate B cells are involved in dermal fibroblast activation and collagen deposition in the skin. However, B cell distribution in epidermal and dermal layers is unknown. Here, We aim to characterize the distribution of B cells residing in normal skin and keloidal scars. METHODS/STUDY POPULATION: One abdominal normal skin sample and two keloid samples (ear and shoulder) were obtained from the University of 